\title{
Commentaire : le patrimoine ferroviaire urbain
}

\author{
Marie-Noëlle Polino
}

\section{OpenEdition}

\section{Journals}

Édition électronique

URL : https://journals.openedition.org/rhcf/1827

DOI : 10.4000/rhcf.1827

Éditeur

Rails \& histoire

Édition imprimée

Date de publication : 1 décembre 2003

Pagination : $547-548$

ISBN : 0996-9403

ISSN : 0996-9403

Référence électronique

Marie-Noëlle Polino, "Commentaire : le patrimoine ferroviaire urbain », Revue d'histoire des chemins de fer [En ligne], 28-29 | 2003, mis en ligne le 06 janvier 2015, consulté le 22 avril 2022. URL : http:// journals.openedition.org/rhcf/1827 ; DOI : https://doi.org/10.4000/rhcf.1827 


\section{Marie-Noëlle POLINO}

\section{Commentaire : le patrimoine ferroviaire urbain}

Les trois chercheurs dont les communications qui précèdent présentent les travaux de doctorat ont pour points communs : pour deux d'entre eux, d'avoir été soutenus par une bourse de l'association ; pour tous les trois, d'avoir contribué pour le moins au signalement, au sauvetage et, pour M. Nogue, au dépôt dans les archives publiques des fonds qu'ils ont mis au jour pendant leurs travaux. Les objectifs de l'AHICF - susciter et appuyer de nouvelles recherches, encourager la préservation du patrimoine - sont là présents, et atteints. Le numéro 23 de la Revue d'histoire des chemins de fer, revue de l'AHICF, publie déjà des études de MM. Le Bollan et Laugier présentées dans le cadre du groupe de recherche «Chemins de fer, architecture et villes », auquel M. Nogue avait également participé.

Je me risque à trois commentaires qui vont permettre de rapprocher ces trois cas que nous avons choisi à dessein très différents par les lieux et les temps.

Les trois recherches présentées nous content l'histoire d'emprises ferroviaires urbaines, qu'il s'agisse des dépôts et ateliers de Rennes et de Marseille et, parmi les réalisations de Laffaille, des rotondes de Béthune et de Metz. Mais le lien avec la ville n'entre pas comme un facteur déterminant dans l'évolution des sites sinon à titre de contrainte externe qui est imposée aux concepteurs de leur construction et de leur organisation. Il est tardif et n'intervient qu'au moment de la reconversionréhabilitation, le cas échéant. C'est paradoxalement à ce moment que le caractère urbain des équipements décide de leur survie.

Ce qui prévaut en revanche, ce sont des logiques économiques, internes à l'activité ferroviaire, et qui se déploient à l'échelle des réseaux, celles d'une grande région ou de l'espace national et qui seules expliquent la physionomie des sites, du bâti et la consistance des héritages. Nous voyons ici l'effet des méthodes d'organisation du travail dans l'espace et dans son articulation, dans les distances et les cheminements et circulations internes aux sites, dans les formes architecturales et le paysage urbain, dans les formes urbaines aussi, comme nous y voyons la marque des fluctuations du volume de l'activité ou de sa nature technique. 
Les trois recherches, c'était le souhait de notre appel à communication, touchent à l'architecture et à la problématique du patrimoine c'est-à-dire posent la question des valeurs et des jugements qui les prennent pour critères : valeur architecturale, valeur patrimoniale. La réponse, les trois auteurs le montrent bien, est dans une analyse exacte, minutieuse et sans préjugés des bâtiments et des sites comme donnés : ils sont le résultat d'une création qu'il importe de restituer mais aussi porteurs d'une identité formelle qui seule souvent survit à une fonction perdue et qui permet une éventuelle reconversion.

On voit combien les notions qui ont longtemps animé seules les débats sur les bâtiments à destination technique - «patrimoine industriel » ou non ? « bâtiments fonctionnels » et non bâtiments tout court, bâtiments-types mieux étudiés sur plan qu'autrement... - ont fait écran à une connaissance précise de leur identité et de leurs possibilités.

Cependant, quelles que soient la qualité de l'analyse et la richesse des connaissances, nous rejoignons Nicolas Nogue dans son analyse de la survie des 19 rotondes qu'il a étudiées, quand il définit ainsi les conditions à la conservation de ce type de patrimoine par reconversion. Elles paraitront brutales et sélectives, mais sont confirmées par l'expérience :

- un « tissu urbain stimulant»

- «le poids significatif de la rotonde (ou du site ferroviaire en général), devenue monument, dans la culture locale » (on l'a vu avec le sauvetage récent de la rotonde de Chambéry)

- une « qualité architecturale à la fois monumentale et intrigante qui les signale avec force dans le contexte urbain ». 